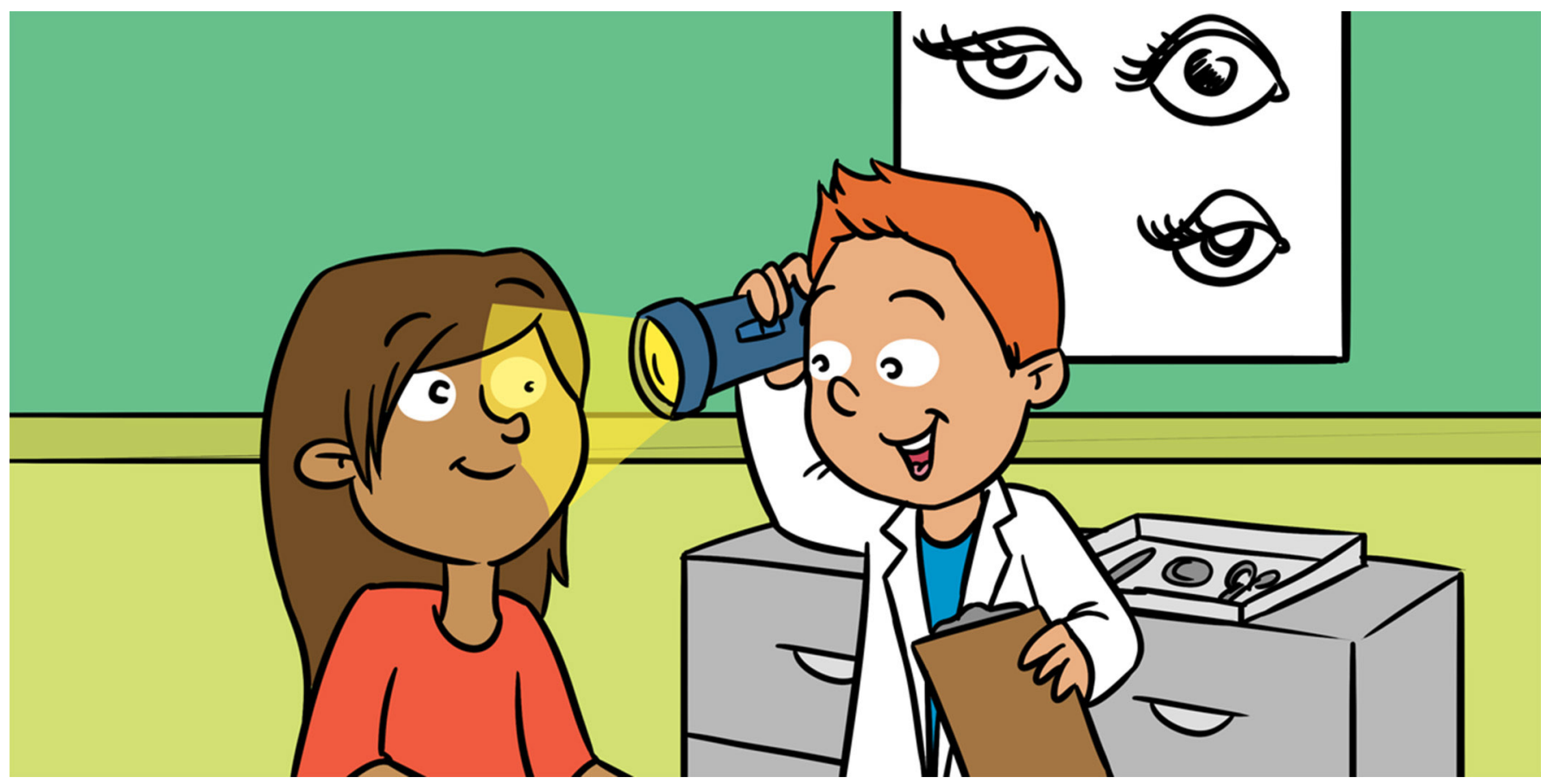

\title{
PUPILS: A WINDOW INTO THE MIND
}

\section{Alexis Torres and Michael C. Hout*}

Psychology Department, New Mexico State University, Las Cruces, NM, United States

YOUNG REVIEWER:

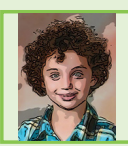

Your pupil, the opening at the center of your eye, allows you to gather information from the world around you. But a lesser known fact about the pupil is that by closely examining how it constricts (gets smaller) and dilates (gets larger) in response to certain stimuli, scientists can gain crucial insights about what is going on in the brain. When the environment is bright, the pupil constricts, and when it is dim, the pupil dilates. But environmental responses-like the response to light-are just the tip of the iceberg. The pupils also respond when people ingest certain drugs, when they feel strong emotions, when they retrieve a memory, or when they are concentrating or thinking very hard about something. In this article, we will discuss how pupils function, how they respond to environmental stimuli, and how their reactions are reflective of cognitive processing-that is, the inner workings and computations going on in the mind. As you will discover, the small opening at the center of your eyes can tell us a whole lot about what is happening in your brain! 
PUPIL

The opening at the center of the iris that allows light to enter the eye.

\section{Figure 1}

An analogy can be made between a camera and the eye. The iris (shown in green at the front of the eye, surrounding the pupil) is similar to the camera's diaphragm (shown in green at the front of the camera lens) and the pupil (the small opening at the center of the eye) is similar to the camera's aperture (the black opening at the center of the camera lens that allows light in). The iris/ diaphragm adjusts the size of the pupil/ aperture to allow the appropriate light into the eye/camera. The light is absorbed/ captured at the back of the eye/camera by the retina/film (shown in purple at the back of the eye, and in purple on the camera, behind the lens).

The opening at the center of your eye allows light to enter it, enabling you to gather information about the world around you, but the opposite is true, too-insights can be gained about what is going on in your brain based on the behavior of your pupils. The pupil is the opening at the center of the eye that appears as a black dot surrounded by the colored part of the eye, the iris. The iris is a muscle in the eye that functions like the diaphragm of a camera (see Figure 1). The iris responds to the amount of light entering the eye by adjusting the size and diameter of the pupil (the aperture), in order to allow the appropriate amount of light into the eye (the camera). Light travels through fluid in the eye and is then absorbed at the back of the eye, in an area known as the retina. The retina is covered in specialized cells called photoreceptors (think of this part of the eye as the film of a camera where the picture is captured). Photoreceptors gather information from the light and send it to the brain to be processed into the image you see.

\section{HOW DOES THE PUPIL WORK?}

When lighting is intense, the iris responds by contracting (flexing) - making the pupil smaller and thereby allowing less light into the eye. When lighting is dim, the iris responds by dilating (relaxing) - making the pupil larger for situations in which more light is needed to see. This is called the pupillary light reflex [1]. The autonomic nervous system-the part of the nervous system responsible for unconscious and involuntary processes, which are processes that we cannot consciously control, like heartbeat and digestion-is also responsible for the pupillary light reflex. Pupillary responses, then, can partly be understood as involuntary or unconscious physical responses to lighting conditions, but this is actually just the tip of the iceberg! (see Figure 2).

The pupillary response as described above is a bottom-up process. We can think of bottom-up processing as processing information (that is, light, sound, pressure, heat, or chemicals) from the body "up" to the brain.
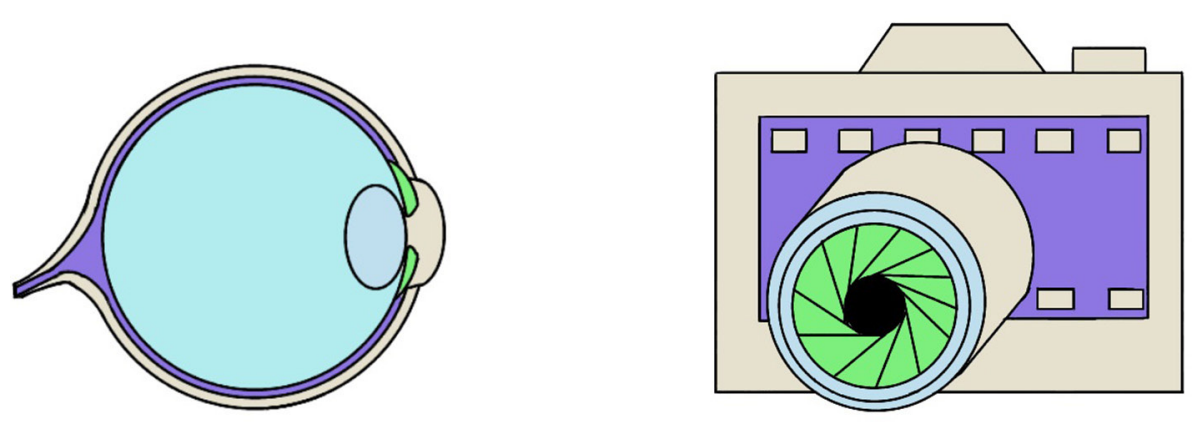

Figure 1 
Figure 2

In this figure, you see the same person's pupil in three different states. The pictures were taken using an eyetracker; blue coloration indicates the location and size of the pupil. In the center panel, you see the person's pupil at rest, after being exposed to normal indoor lighting conditions. In the left panel, you see the pupil constricted (decreased in size) after exposure to a bright flashlight. In the right panel, you see the pupil dilated (increased in size) following a period in which the person sat in a dark room.

\section{PUPILLOMETRY}

The study and measurement of the diameter of the pupil and how it reacts to environmental stimuli and mental processes.

\section{COGNITIVE LOAD}

The amount of mental effort used during information processing.

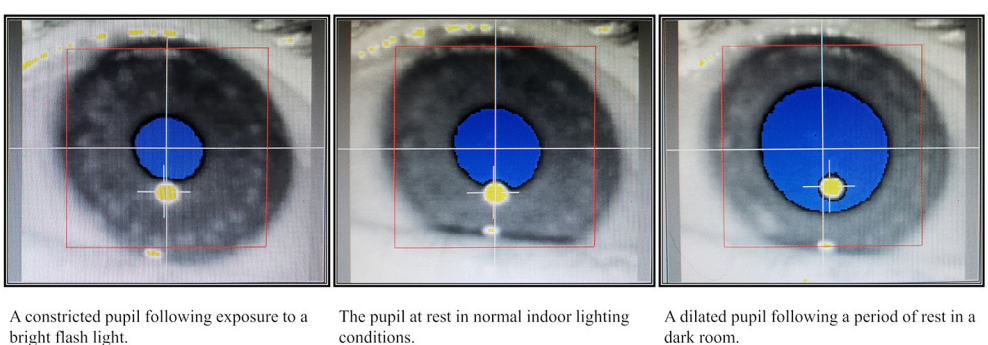

Figure 2

In bottom-up processing, information enters the body by way of the sensory organs (eyes, ears, skin, mouth, nose): "the bottom." Information is then taken up to and processed in the brain, "the top," to create an interpretation (that is, a perception) of the information, a memory of it, or other higher-level thinking. Pupillary responses to light are considered bottom-up processing because they result from the external stimulus (a certain intensity of light entering the eye) being processed in the brain and then perceived (the intensity of the light is perceived as brightness). From there, the brain sends signals back to the iris, causing it to contract or dilate, depending on the brightness of the light [2]. It seems to make sense, then, that the pupil should only respond to lighting conditions because controlling the amount of light seems to be the pupil's primary purpose. Surprisingly, this is not entirely true. By studying pupillary responses, we now know that pupils can tell us about much more complex activity that is going on in the brain.

\section{PUPILS TELL US ABOUT MORE THAN BRIGHTNESS}

Pupillometry is a method used to study pupillary responses by measuring changes in the size of the pupil in response to various types of stimulation. Pupillometry has revealed an additional top-down relationship between pupillary responses and several mental operations that are sometimes quite complex [1]. Top-down processing can be thought of as higher-level information from the brain that is sent "down" to the body. In the rest of this article, we will tell you about the exciting and unexpected relationship between the opening of your eye and the inner workings of your mind: Specifically, perception, emotional responses, and cognitive load.

\section{PERCEPTION: THINKING ABOUT LIGHT}

Surprisingly, scientists have recently found that pupils even respond to stimuli that imply a lighting condition in the same way they do when we experience real shifts in lighting conditions. Put simply, even when 


\section{DILATION}

A relaxation of the muscles in the iris causing the pupil to become larger in diameter. there is no change in the actual brightness of a stimulus, a person's pupils may respond by constricting because that stimulus usually tells us there are bright lighting conditions. For example, Binda et al. [3] showed participants equally bright images of the sun and the moon, which we know are typically bright or dim, and scrambled images that did not look like anything specific. The images that did not look like anything specific were either "mean luminance" or "phase scrambled," and, unlike the sun and moon images, they were meant to lack meaning so that any pupillary response to them was due to their brightness alone, and not because they implied (or suggested) a lighting condition. Mean luminance images were uniform in color and matched the average overall brightness and color of the entire image of the sun. The phase scrambled images of the sun can be thought of as a jigsaw puzzle, with all of the pieces mixed up. The phase scrambled images were also the same average overall brightness as the original sun images, but the pieces of the image were scrambled so that the picture no longer looked like an image of the sun.

In this way, the four types of images differed in their meaningfulness but not their brightness, so any difference in pupil dilation that was observed would have to result from something other than differences in brightness. The researchers found that individuals' pupils reacted to images of the sun (which is normally quite bright) by contracting. However, images of the moon and the images that did not look like anything specific did not make the participants' pupils contract, even though they were just as bright as the picture of the sun! Likewise, Mathôt et al. [4] found that participants' pupils contracted when presented with words (both spoken and visual) that merely conveyed the concept of light, and that their pupils dilated for words conveying darkness. It seems that understanding or thinking about the concepts of light or darkness can sometimes be enough to cause the same response in the pupils as actual light and darkness cause. This tells us that the pupils can respond not only to environmental, bottom-up information, but can also be stimulated by top-down processes, or information coming from the brain instead of the environment.

\section{EMOTION: YOU CAN SEE IT IN YOUR EYES}

Brain structures that are involved in higher-level processes, such as emotion and cognition (thinking), are also known to stimulate pupillary activity. Activity in the regions of the brain that help us feel emotions can also increase pupil dilation. Things in the environment that cause us to have emotional responses, either positive or negative, can result in pupillary dilation. For example, Partala and Surakka [5] presented participants with a series of sounds and monitored the participants' 
pupils while they listened. After each presentation, participants rated the sounds as emotionally positive, negative, or neutral. Sounds that were rated as positive (such as a baby laughing) or negative (such as a baby crying, or a couple fighting) resulted in increases in pupil dilation compared with neutral sounds (such as background office noises), which had little effect on pupil size.

\section{COGNITION: THINKING HARD SHOWS}

If you try to do something different, something that you have not done before, more mental effort is required to perform that task, meaning that you have to think harder. Increased pupillary dilation also results from this increase in mental effort, which is often referred to as cognitive load. When the increase in cognitive load lasts for a while, dilation of the pupils also lasts for a while, signaling that the person is continuing to think about the difficult task and pay attention to it. Increased cognitive load is thought to be associated with areas of the brain responsible for continuous attention, located in the frontal lobe of the brain (behind the forehead). This pattern of increased pupil dilation resulting from increased cognitive load is seen when individuals perform difficult tasks. Tasks such as doing difficult math problems, memorizing large sets of information, or counting backwards by increments of seven cause an increase in cognitive load and therefore produce increased pupillary dilation [2]. ${ }^{1}$

Using pupil dilation as an indicator of increased cognitive load, studies on memory have found that cognitive load increases when memorizing, remembering, and recognizing information, and cognitive load is greater for more difficult content. For example, Papesh et al. [6] found that cognitive load increased significantly when participants were asked to memorize and retrieve made-up words (like "garp") from memory, as compared with real words. The researchers found that, when more mental effort was used to memorize information, that information was remembered better later on. More importantly, this was also reflected in pupillary dilation; the stronger the memory, the more dilated one's pupils tended to be.

\footnotetext{
${ }^{1}$ You can test all of this for yourself: Have a friend agree to keep eye contact with you (this may be the most challenging part, since it is a little awkward), then ask him/ her easy math questions (What is $2+2,1+1 \ldots$ ?). You should not notice a change in dilation. Now, ask a difficult math question (Like, what is 53 * 87?), and you might notice a sudden increase in dilation. If your friend can figure out the problem or stops trying to solve it, you may notice his/her pupils will return to normal.
} 
CONTRACTION

A tightening of the muscles in the iris causing the pupil to become smaller in diameter.
These findings also apply to children's ability to use short-term memory-the memory system used when trying to remember information for a short period of time. Imagine, for instance, that you hear an ice cream truck and generously offer to buy three of your friends a snack. If you try to remember what kind of treat each of them wants so you can run over and buy it for them, you are using your short-term memory to hold onto that information. Johnson et al. [7] studied short-term memory using a task in which participants memorized long sequences of numbers and immediately reported them back. The researchers watched the participants' pupils for increased and sustained dilation, which told them when the participants were memorizing information and when they were not. After memorizing six numbers, children's pupils typically began constricting, but adult's pupils stayed dilated. This indicated that, at six numbers, children hit their memory limit and stopped memorizing, but adults did not. This pupillometry study confirmed that children have a smaller short-term memory capacity than adults have.

What is different about pupillary responses that happen during cognitive load and pupillary responses that happen during perception and emotion is that both pupil contraction and dilation result from cognitive load. The pupils dilate when information is being processed or remembered, since both are cognitively demanding tasks. The pupils then often constrict upon reporting the information, because cognitive load decreases once the information has been successfully remembered. Finally, pupil size returns to intermediate size when mental processing is complete [2]. The monitoring of pupil dilation, then, is useful for tracking when we are putting more effort into thinking, from when we start processing information to when we eventually report the information.

\section{CONCLUSION}

Pupil dilation is, perhaps, an unusual way to understand the human mind, but it is clearly a useful one. This method could even potentially be used to study different populations, such as babies, children, individuals with mental disorders, or those with difficulties communicating. Studying the pupil allows us to take advantage of the fact that the eye is an extension of the brain. As Eckard Hess [8] put it, "it is almost as though a portion of the brain were in plain sight for the psychologist to peer at."

\section{REFERENCES}

1. Sirois, S., and Brisson, J. 2014. Pupillometry. Wiley Interdiscip. Rev. Cogn. Sci. 5:679-92. doi: 10.1002/wcs.1323 
2. Steinhauer, S. R., Siegle, G. J., Condray, R., and Pless, M. 2004. Sympathetic and parasympathetic innervation of pupillary dilation during sustained processing. Int. J. Psychophysiol. 52:77-86. doi: 10.1016/j.ijpsycho.2003.12.005

3. Binda, P., Pereverzeva, M., and Murray, S. O. 2013. Pupil constrictions to photographs of the sun. J. Vis. 13:8. doi: 10.1167/13.6.8

4. Mathôt, S., Grainger, J., and Strijkers, K. 2017. Pupillary responses to words that convey a sense of brightness or darkness. Psychol. Sci. 28:1116-24. doi: 10.1177/0956797617702699

5. Partala, T., and Surakka, V. 2003. Pupil size variation as an indication of affective processing. Int. J. Hum. Comput. Stud. 59:185-98. doi: 10.1016/S10715819(03)00017-X

6. Papesh, M. H., Goldinger, S. D., and Hout, M. C. 2012. Memory strength and specificity revealed by pupillometry. Int. J. Psychophysiol. 83:56-64. doi: 10.1016/j.ijpsycho.2011.10.002

7. Johnson, E. L., Miller Singley, A. T., Peckham, A. D., Johnson, S. L., and Bunge, S. A. 2014. Task-evoked pupillometry provides a window into the development of short-term memory capacity. Front. Psychol. 5:218. doi: 10.3389/ fpsyg.2014.00218

8. Hess, E. H. 1965. Attitude and pupil size. Sci. Am. 212:46-54. doi: 10.1038/ scientificamerican0465-46

SUBMITTED: 17 June 2018; ACCEPTED: 04 January 2019; PUBLISHED ONLINE: 06 February 2019.

EDITED BY: Alessandro Antonietti, Catholic University of Sacred Heart, Italy

CITATION: Torres A and Hout MC (2019) Pupils: A Window Into the Mind. Front. Young Minds 7:3. doi: 10.3389/frym.2019.00003

CONFLICT OF INTEREST STATEMENT: The authors declare that the research was conducted in the absence of any commercial or financial relationships that could be construed as a potential conflict of interest.

COPYRIGHT @ 2019 Torres and Hout. This is an open-access article distributed under the terms of the Creative Commons Attribution License (CC BY). The use, distribution or reproduction in other forums is permitted, provided the original author(s) and the copyright owner(s) are credited and that the original publication in this journal is cited, in accordance with accepted academic practice. No use, distribution or reproduction is permitted which does not comply with these terms.

\section{YOUNG REVIEWER}

\section{JACOB, AGE: 11}

My favorite sports to play are soccer, baseball, basketball, and tennis. My favorite car company is Tesla, which makes electric cars. My favorite fast food restaurant is Burger King. My favorite slow food restaurant is Moon Star Chinese restaurant. 
I weigh 68 pounds. I am nice looking and nice to people. I love political comedians because they teach you about the world and make you laugh at things that would otherwise be painful.

\section{AUTHORS}
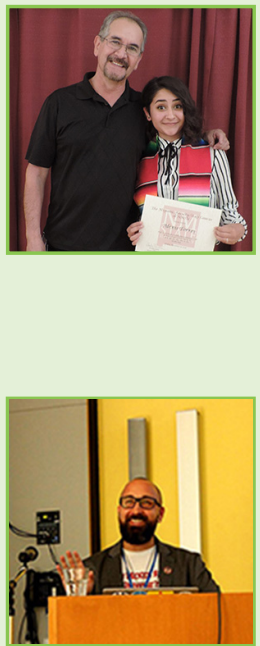

\section{ALEXIS TORRES}

I am a lab-tech in the Serrano Neurobiology Lab at New Mexico State University. I have dedicated much of my undergraduate career to research and hope to continue on that path. Having just earned my Bachelor's Degrees in Psychology and Philosophy, I am eager to move forward as I apply for cognitive neuroscience PhD research programs. In my free time, I enjoy hiking, binge watching TV series, reading books, and playing with my precious beagle terrier.

\section{MICHAEL C. HOUT}

I am an Associate Professor in the Psychology Department at New Mexico State University, and an Associate Editor at the journal Attention, Perception, \& Psychophysics. My research examines many different things, but I primarily study visual search (how people find things) and eye movements (where and why we move our eyes). In my limited spare time, I like to play with my dogs, go on motorcycle rides, hike, travel, and play hockey. ${ }^{*}$ mhoutanmsu.edu 